\title{
Spatio-temporal dynamics of EEG features during sleep in major depressive disorder after treatment with escitalopram:A pilot study
}

Li Wu

Hangzhou Dianzi University

Xue-Qin Wang

Peking University Sixth Hospital

Teng-Fei Dong

Hangzhou Dianzi University

Ling Lei

Hangzhou Dianzi University

Su-Xia Li

Peking University Sixth Hospital

\section{Qi-Qi Cheng}

Hangzhou Dianzi University

Yong Yang ( $\sim$ yyang@hdu.edu.cn )

Hangzhou Dianzi University

\section{Research article}

Keywords: EEG, major depressive disorder, escitalopram, power spectrum, nonlinear dynamics, spatiotemporal dynamics

Posted Date: November 7th, 2019

DOI: https://doi.org/10.21203/rs.2.16941/v1

License: (c) (7) This work is licensed under a Creative Commons Attribution 4.0 International License. Read Full License 


\section{Abstract}

Background: Previous studies have shown escitalopram is related to sleep quality. However, effects of escitalopram on dynamics of electroencephalogram (EEG) features especially during different sleep stages have not been reported. This study may help to reveal pharmacological mechanism underlying escitalopram treatment.

Methods: The spatial and temporal responses of patients with major depressive disorder (MDD) to escitalopram treatment were analyzed in this study. Eleven MDD patients and eleven healthy control subjects who completed whole tests were included in the final statistics. Six-channel sleep EEG signals were acquired during sleep. Power spectrum and nonlinear dynamics were used to analyze the spatiotemporal dynamics features of the sleep EEG after escitalopram treatment.

Results: For temporal dynamics: after treatment, there was a significant increase in the relative energy (RE) of band, accompanied by a significant decrease in the RE of band. Lempel-Ziv complexity and CO complexity values were significantly lower. EEG changes at different sleep stages also showed the same regulation as the whole sleep process. For spatio dynamics: after treatment, the EEG response of the left and right hemisphere showed asymmetry. Further analysis of brain region-specific targets found that the frontal cortex showed a more intense EEG response with escitalopram treatment than central and occipital cortices.

Conclusions: These findings may contribute to a comprehensive understanding of the pharmacological mechanism of escitalopram in the treatment of depression.

\section{Background}

Major depressive disorder (MDD) is one of the most common diseases worldwide and is responsible for premature deaths and disability (Baghai et al. 2018). In the past, MDD patients have been treated mainly with traditional drugs such as monoamine oxidase inhibitors, or tricyclic and heterocyclic antidepressants (Steve et al. 2003; Hillhouse and Porter 2015). These traditional drugs have some disadvantages such as weak tolerance, large adverse effects and slow action onset. Escitalopram is a highly selective SSRI and therapeutically active S-enantiomer of citalopram, it has been widely used and recommended by clinicians worldwide(Azorin et al. 2004).Compared to other antidepressants, escitalopram is tolerated better, has fewer adverse effects and has a faster onset of action (Sanchez et al. 2014; Azorin et al. 2004; Kennedy et al. 2009; Montgomery et al. 2007). However, its pharmacological mechanism has not yet been clarified completely.

Previous study has shown escitalopram can improve sleep quality in MDD patients (Kostyalik et al. 2014), and was efficacious in treating depressive symptoms in depressed patients suffering from poor sleep quality, and this beneficial effect appeared to be independent of the severity of the patient's sleep problems(Lader et al. 2005). Furthermore, it has been reported that escitalopram is advantageous in the treatment of the core symptoms of MDD, including sleep disturbance (Stein and Lopez 2011). Sleep is a 
complicated process, and can be divided into different stages both temporally, and spatially, and is related to multiple interactions between different brain regions. Therefore, it is necessary to explore the pharmacological mechanism underlying the action of escitalopram, and to investigate the relationship between escitalopram and sleep in both temporal and spatial dimensions. However, until now, there have been few studies involving both temporal and spatial dimensions, only research involving the eye close and open condition (Baskaran et al. 2018). Currently there are questions still to be resolved, such as whether escitalopram acts the same at each sleep stage, or is specific to certain stages. Also it remains unclear as to whether escitalopram is brain region specific such as the frontal, central, or occipital cortices or displays asymmetries in brain hemispheres.

Electroencephalography (EEG) is a suitable option as a neurophysiological biomarker, and displays several advantages, including higher temporal resolution, non-invasiveness, ease of access and low cost (Olbrich and Arns 2013; Baskaran et al. 2012). As a result this method has been widely used for the biomedical investigation of several mental illnesses including MDD, Alzheimer's disease and others (Hunter et al. 2007; Abasolo et al. 2006; Alhaj et al. 2011; Babiloni et al. 2016). EEG signals can be analyzed by linear and non-linear dynamic analyses. Linear analysis such as power spectrum is commonly used to extract the features of sleep EEG signals. Spectral characteristic parameters can reflect the energy information transported by each frequency band. Because the brain is a complex nonlinear system, the use of non-linear dynamic analysis may also be used to reflect brain states accurately (Janjarasjitt et al. 2008; Kang et al. 2015; Mikšovský and Raidl 2006). Among the non-linear features, complexity is suitable as it can be calculated within a short time series and fast speed.

In the present study, we investigated the spatio-temporal dynamics of sleep EEG features before and after escitalopram treatment. Both linear and non-linear dynamic analyses may provide a more comprehensive understanding of the pharmacological mechanism of escitalopram in the treatment of depression.

\section{Methods}

\subsection{Participants}

A total of 58 subjects participated in the study, which included 30 MDD patients and 28 healthy controls. However, more than half of MDD patients were excluded from the final analysis because their electrode became detached, more than half of healthy controls were eliminated because their sleep time was less than 5.5 hours on the experimental night. Finally, 11 healthy male adult controls and 11 male MDD patients completed the study. The MDD patients ages' ranged from 22 to 40 years (meanSD: $30.64 \pm 5.52$ years).These patients reported no history of any other psychiatric disorder or prior take of antidepressants. All patients, were from The Peking University Sixth Hospital, and met the criteria for major depression defined in the Diagnostic and Statistical Manual of Mental Disorders, 4th edition (DSMIV)(Do 2011). Diagnosis was established by experienced psychiatrists using the Structured Clinical Interview for DSM-IV disorders (SCID). A minimum score of 22 points on the 17 item Hamilton Depression Scale (HRSD - 17) (Snaith 1977) was required to be enrolled in the study. Professional scorers from the 
Peking University Sixth Hospital conducted the HRSD measurements two times. The first time occurred before the treatment of escitalopram, and the second time occurred on day 57 of escitalopram treatment. The exclusion criteria included: (1) age $<18$ or $45>$ years, (2) presence of additional psychotic symptoms, (3) cognitive impairment or personality disorders, (4) history of other mental illness, (5) suicidal ideation or behaviors.

Control participants included 11 physically and mentally healthy male volunteers whose ages were between 22 and 38 years (mean SD: $27.72 \pm 4.79$ years). The inclusion criteria included: (1) absence of psychiatric illnesses diagnosed by the DSM-IV criteria, (2) a maximum score of 7 points on the 17 - item HRSD (3) a maximum score of 7 points on the 14 - item Hamilton Anxiety Scale (HAMA) (Maier et al. 1988), (4) no current or pass chronic physical diseases (e.g., cardiovascular disease, diabetes, rheumatoid arthritis, et al.), (5) non-shift worker.

All of the participants were Han Chinese. They signed written informed consent forms before participation. The study was approved by the ethics committee of Peking University Sixth Hospital, Beijing, China, in accordance with the Helsinki Declaration.

\subsection{Polysomnographic recording}

All the depressive patients underwent polysomnographic recording two times. The first time conducted before the treatment of escitalopram, and the second time conducted on day 57 of escitalopram treatment.

Overnight polysomnographic recording included electroencephalography (EEG; including F3, F4, C3, C4, 01, and 02, with reference to the contralateral mastoid; International10 - 20system), electrooculography (EOG), electromyography (EMG), and electrocardiography (ECG). The signals were digitized at a sampling rate of $256 \mathrm{~Hz}$, and an electrode impedance $<5 \mathrm{~K} \Omega$. Thirty - second epochs were used for manual analysis, and sleep stages were scored offline according to the criterion of the American Academy of Sleep Medicine (AASM), using the standard polysomnographic sleep recordings.

\subsection{EEG signal processing}

In the processing environment of MATLAB R2016b, using EEGLAB toolkits (University of California San Diego), power frequency interference was eliminated by using a $50 \mathrm{~Hz}$ notch, and data was filtered from 0.5 to $30 \mathrm{~Hz}$ by using band pass filter (Delorme and Makeig 2004). Each sample had corresponding sleep staging files. However, because the sample duration data was too large, and some data frames had large artifacts, we chose the entire artifact - free frames (30 seconds) from every sleep type (including Wake, rapid-eye-movement (REM), NREM1, NREM2 and NREM3) according to sleep staging files.

The EEG signal processing includes two aspects: linear analysis and nonlinear dynamic analysis. 


\subsubsection{Linear analysis囚Power spectrum}

The power spectrum reflects the energy information carried by the brain waves in each frequency band. According to the frequency, the EEG signals are divided into several categories: $\delta 1(0.5-2 \mathrm{~Hz}), \delta 2$ (2 $4 \mathrm{~Hz}), \theta(4-8 \mathrm{~Hz}), a(8-13 \mathrm{~Hz}), \beta 1(13-20 \mathrm{~Hz})$ and $\beta 2(20-30 \mathrm{~Hz})$. In the present study, each frequency band power was obtained by using fast Fourier transform (FFT) analysis (Faust et al. 2008; Pardey et al. 1996b; Welch 1988). FFT calculation was performed on 3 second non-overlapping consecutive window (Hamming window). The average values of the different sleep stages were computed form the 30 seconds of data obtained previously. In order to reduce specific individual differences, the relative energy (RE) was computed. The RE corresponds to the ratio between the power value of each frequency band and the sum of the power values in the following calculation:

[Please see supplementary files for formula]

\subsubsection{Nonlinear dynamic analysis: LZC, COC}

Correlation dimension, complexity, entropy and Lyapunov exponents are common non-linear features in EEG signal analysis. Correlation dimension and Lyapunov exponents require large data sets and strict dimensional measurements which are not suitable for EEG analysis. Whereas Lempel-Ziv Complexity (LZC) and CO Complexity ( $\mathrm{COC}$ ) are more suitable, because they require small datasets and have high computation speeds. Therefore, in the present study, LZC and COC were used to characterize the sleep state of patients with MDD. LZC represents the rate of appearance of a new pattern in a time series from a one dimensional perspective ( $\mathrm{Li}$ and Wang 2008). A ratio of the area of the disorder component over the area of the original time series is considers as a complexity measurement, which is denoted as $\mathrm{CO}$ (Chen et al. 2000). The higher the LZC, the more likely it is that a new model will appear, highlighting complex dynamic behavior. The higher the $\mathrm{COC}$, the more probability there is that random motion may appear.

In order to obtain 28 seconds sequences, the first and last second of each of them were removed from the 30 seconds previously selected sequences. This shortened sequence was then divided into 7 segments of 4 seconds each for targeted analysis. For each of those time-windows we considered as important EEG features, the mean of the values by itself as well as the characteristic of values according to the sleep stage.

\subsection{Statistical analysis}

All analyses were performed using the SPSS Statistics version 22.0. We used the paired-samples t-test to investigate the changes in the EEG characteristic parameters between the baseline and the final session, and the independent-sample t-test to compare the results from the patients with MDD and healthy control subjects. We then used the paired-samples t-test to analyze differences between the left and right hemispheres of the cortex. The differences of EEG characteristics in different brain regions (frontal, 
central, occipital) between baseline and final were analyzed by one-way ANOVA. Differences were considered significant when $P<0.05$.

\section{Results}

\subsection{EEG response before and after escitalopram treatment of the whole sleep process}

We investigated the EEG response for MDD patients (before and after escitalopram treatment) and healthy control subjects in the spatial and temporal dimensions. For temporal dynamic analysis, firstly, we compared the RE of different EEG frequency bands of the whole sleep process between MDD patients (before and after escitalopram treatment) and healthy control subjects. Figure 1 ( $\mathrm{\&}$ b) indicate that after treatment, the RE of the $\delta 1$ band was significantly higher than that before treatment $(t(10)=-2.397, p=$ 0.028). The RE of the $\beta 2$ band in patients with MDD before treatment with escitalopram was significantly higher than that in controls $(t(20)=2.513, p=0.045)$, it was significantly decreased to control level after treatment $(t(10)=2.513, p=0.045)$. Additionally, the RE of other frequency bands in MDD patients also had some improvement after treatment, but not statistically significant.

Secondly, we analyzed whether there existed differences in non-linear LZC and COC values during the whole sleep process for MDD patients (before and after escitalopram treatment) and healthy control subjects. As detailed in Fig. 1 (c \& d), the LZC values in patients before treatment with escitalopram was higher than that in controls $(t(20)=2.963, p=0.010)$, it was significantly decreased to control level after treatment $(t(10)=2.626, p=0.030)$. Furthermore, the COC values showed the same regulation as LZC values, the $\mathrm{COC}$ values in patients before treatment with escitalopram was higher than that in controls $(t$ $(20)=2.397, p=0.028)$, it was significantly decreased to control level after treatment $(t(10)=2.862, p=$ $0.007)$.

Fig. 1 about here

\subsection{EEG response before and after escitalopram treatment at different sleep stages}

We compared the changes in RE of each frequency bands at different sleep stages in MDD patients (before and after escitalopram treatment) and healthy controls. Firstly, we compared the RE of each frequency bands during different sleep stages. As shown in Fig. 2a, a significant increase in the RE of $\delta 1$ band in the NREM1 $\left(t\left(_{10}=-2.239, p=0.049\right)\right.$, NREM2 $\left(t\left(_{10}=-2.923, p=0.015\right)\right.$, and REM $\left(t\left(_{10}\right)=-\right.$ $2.648, p=0.024)$ sleep stages after treatment with escitalopram was seen. Additionally, a significant decrease in the RE of $\delta 2$ band $\left(t\left(_{20}=-2.371, p=0.027\right)\right.$, and $\theta$ band $(t(20)=-2.923, p=0.004)$ in the NREM1 sleep stage in patients with MDD before treatment compared with controls (Fig.2b and $2 c$ ).. A 
significant decrease of the $\beta 2$ band in the REM $(t(10)=3.126, p=0.011)$ sleep phase in patients with MDD after treatment compared with before treatment (Fig.2f).

Secondly, we analyzed for differences in non-linear LZC and COC values at different sleep stages in MDD patients and the healthy control subjects. As shown in Fig.2g, LZC values in patients with MDD after treatment decreased significantly compared with that before treatment during NREM1 $\left(t{ }_{10}=3.946, p=\right.$ $0.003), \operatorname{NREM} 2\left(t\left(_{10}\right)=3.527, p=0.005\right)$ and REM $\left(t\left(_{10}\right)=2.920, p=0.015\right)$ sleep stage. While during NREM2 sleep stage LZC values in patients with MDD before treatment increased significantly compared with that in controls $\left(\mathrm{t}_{(20)}=2.847, p=0.011\right)$. As for the COC values, during NREM2 sleep stage showed a significant increase in patients with MDD before treatment compared with controls $\left(t f_{10)}=2.387, p=\right.$ $0.029)$ and a significantly decreased after compared with before treatment $(t(10)=3.126, p=0.011)$ in patients with MDD (Fig.2h).

Fig.2 about here

\subsection{EEG response before and after escitalopram treatment in left and right hemispheres}

For spatio dynamic analysis, we found differences in EEG features between left and right hemispheres in MDD patients (before and after escitalopram treatment). Firstly, we analyzed for differences in the RE between left and right hemispheres during different sleep stages. As shown in Fig. 3a, the RE of the $\delta 1$ band during NREM2 sleep stage in the right hemisphere increased significantly compared with that in the left hemisphere $\left(t_{(10)}=-2.626, p=0.030\right)$ after escitalopram treatment. In Fig. 3c, during the NREM1 sleep stage, the RE of the $\theta$ band in the left hemisphere was significantly higher than that in the right hemisphere $\left(t_{(10)}=-2.626, p=0.017\right)$ after escitalopram treatment. In Fig. $3 f$, during the REM stage, the $\beta 2$ band in the right hemisphere showed a significant decrease compared with that in the left hemisphere $\left(t\left(_{10)}=2.302, p=0.05\right)\right.$ after escitalopram treatment.

Secondly, we also explored differences in non-linear LZC and COC values between left and right hemispheres during different sleep stages. As shown in Fig. 3g, during the REM sleep stage, the LZC values did not differ between the left and right hemispheres before treatment. However, a significant decrease in the LZC value in the right hemisphere compared with that in the left $(t(10)=4.632, p=0.001)$ was found after escitalopram treatment.

Fig.3 about here

\subsection{EEG response among different brain regions}

Since escitalopram treatment may have brain region - specific effects, we investigated the EEG response in different cortical areas (frontal, central and occipital) in MDD patients (before and after treatment). 
We found significant changes in the frontal cortex in NREM1, NREM2 and REM sleep stage after treatment. For NREM1, we found that the $\delta 1$ band RE significantly increased $(F(2,30)=6.961, p=0.003)$ in the frontal cortex compared with that in the central and occipital cortices, which illustrated by warmer colors in the frontal lobe. In the same sleep stage, the $\beta 2$ band $\operatorname{RE}(F(2,30)=3.928, p=0.031)$ and the nonlinear LZC values $(F(2,30)=6.176, p=0.006)$ in the frontal cortex significantly decreased compared with that in the central and occipital cortices, which illustrated by colder colors in the frontal cortex.

For the NREM 2 and REM sleep stages, the $\delta 1$ band RE significantly increased in the frontal cortex, (NREM2: $F(2,30)=6.863, p=0.004)$; REM: $(F(2,30)=5.740, p=0.008)$, which illustrated by warmer colors in the frontal lobe. These results showed a more intense EEG response in the frontal cortex than that in any other brain regions.

Fig.4 about here

\section{Discussion}

With regard to temporal dynamics study, power spectrum results showed that after treatment, the 1 band RE significant increased, whereas the $\beta 2$ band RE significantly decrease. Previous studies have found that the $\delta 1$ band was seen during phases of reduced alertness and sleep (Knyazev 2012). The $\beta 2$ frequency range is thought to reflect behavioral arousal and attention processes (Hlinka et al. 2010; Nofzinger et al. 2000). Therefore, an increase in the $\delta 1$ band and a decrease in the $\beta 2$ band may be consistent with an improved sleep quality.

It is notable that after escitalopram treatment, non-linear LZC and COC values also showed a significant decrease. A decrease in LZC and COC values may correspond to a decrease in brain wave activity and an increase in lethargy. Therefore, the non-linear dynamic features also revealed that escitalopram can improve sleep quality in MDD patients. Until now, there have been few reports looking at changes in nonlinear LZC and COC values before and after escitalopram treatment, especially during the sleep process.

Our spatial dynamic study has found that after escitalopram treatment, sleep EEG responses in the left and right hemispheres were asymmetrical. For the NREM1 sleep stage, the RE of $\theta$ band in the left hemisphere was higher than that in the right. Given the association between $\theta$ band activity and rostral anterior cingulate cortex activity, an asymmetry in $\theta$ activity may reflect rapid escitalopram - induced activity within the default mode network. This in turn may indicate continued re-establishment of fronto cingulate connections, which may be required to relieve depressive symptoms.

For the NREM2 sleep stage, the RE of $\delta 1$ band in the right hemisphere was higher than that in the left after escitalopram treatment. This result is in line with that of Anusha's research looking at power spectrum changes under closed eye conditions. In which Anusha et al. found that escitalopram responders showed greater delta power in the right hemisphere at 2-week of escitalopram treatment (Baskaran et al. 2018). Additionally, right lateralization of delta in escitalopram responders are similar to 
reports of increased slow wave activity in the right hemisphere in MDD patients (Iznak and Sorokin 2013). Therefore, this feature may reflect a subtype of MDD patients that respond well to escitalopram.

For the REM sleep stage, the RE of $\beta 2$ band was lower in the right hemisphere than that in the left after escitalopram treatment. Previous research has found that the EEG beta power has been shown to have a temporal association with cortisol secretion suggesting a mechanistic link between increased hypothalamic - pituitary - adrenal function and higher frequency brain activation (Chapotot et al. 1998). In addition, changes in beta asymmetry observed in the patients after escitalopram treatment may reflect antidepressant induced variations in arousal. Differences in LZC values between the right and left hemispheres in reaction to escitalopram treatment have not yet been explored. In our study, the LZC values of the right hemisphere were lower than that in the left hemisphere during the REM sleep stage after escitalopram treatment. The neurobiological basis of this finding in the context of response to escitalopram treatment is poorly understood, and this finding needs to be further explored and verified in a wider range of studies.

Spatial dynamic research of brain region-specific targets demonstrates that after escitalopram treatment, the frontal cortex showed a more intense EEG response compared with the central, and occipital cortices. Previous research has found that the frontal lobe has a regulatory role in emotional cognition (George et al. 2010). The prefrontal cortex is rich in 5-HT2A receptors, and pharmacological studies have shown that 5-HT2A receptors are involved in antidepressant behaviors (Anna et al. 2015), and that they may play an antidepressant role by increasing the release of $5-\mathrm{HT}$. Therefore, the greater the response of the frontal cortex, may be indicative of a good response to escitalopram treatment in MDD patients.

In summary, the spatio-temporal dynamics of the EEG features during sleep in MDD patients with escitalopram treatment was explored in this study. Our findings may aid in unravelling the mechanisms underlying the action of escitalopram treatment. However, these results were based on a small sample size, and therefore, larger sample size will be needed to verify them for future studies.

\section{Conclusions}

The findings presented within this study are encouraging in several aspects. Firstly, temporal dynamics study demonstrated that there appeared an increase in the $\delta 1$ band, a decrease in the $\beta 2$ band, and a decrease in non-linear LZC and COC values after escitalopram treatment. Secondly, spatial dynamic study indicated that sleep EEG responses in the left and right hemispheres were asymmetrical, the frontal cortex showed a more intense EEG response compared with the central, and occipital cortices. These findings may contribute to a comprehensive understanding of the pharmacological mechanism of escitalopram in the treatment of depression.

\section{Abbreviations}


Electroencephalogram (EEG), Major depressive disorder (MDD), Lempel-Ziv Complexity (LZC), C0 Complexity (COC), Rapid Eye Movement (REM).

\section{Declarations}

Ethics approval and consent to participate: All of the participants were Han Chinese. They signed written informed consent forms before participation. The study was approved by the ethics committee of Peking University Sixth Hospital, Beijing, China, in accordance with the Helsinki Declaration.

Consent for publication: Not applicable.

Availability of data and material: Not applicable.

Competing interests: All authors declare that there are no conflicts of interest associated with this publication.

Funding: This work was supported in part by the National Natural Science Foundation of China (no. 81671038, 61871165, 81171251 and 81871071), Capital's Funds for Health Improvement and Research (no. 2014-4-4113), Beijing Municipal Science and Technology Commission (no. Z161100000516128), and Beijing Municipal Natural Science Foundation (no. 7162101).

Authors' contributions: Li Wu and Yong Yang were responsible for processing and analyzing data, and were major contributor in writing the manuscript; Xue -Qin Wang and Xu -Xia Li made great contributions to experiment and data acquisition, and they also helped in modifying the manuscript; Teng-Fei Dong, Ling Lei and Qi-Qi Cheng wrote the paper. All authors have read and approved the final manuscript.

Acknowledgements: Not applicable.

\section{References}

Abasolo D, Hornero R, Gomez C, Garcia M, Lopez M (2006) Analysis of EEG background activity in Alzheimer's disease patients with Lempel-Ziv complexity and central tendency measure. Med Eng Phys 28: $315-22$

Alhaj H, Wisniewski G, McAllister-Williams RH (2011) The use of the EEG in measuring therapeutic drug action: focus on depression and antidepressants. J Psychopharmacol 25: 1175-91

Anna CÉ, Lucila K, Pau C, Analía B, Francesc A (2015) 5-HT2A receptors are involved in cognitive but not antidepressant effects of fluoxetine. Eur Neuropsychopharmacol 25: 1353-1361

Azorin JM, Llorca PM, Despiegel N, Verpillat P (2004) [Escitalopram is more effective than citalopram for the treatment of severe major depressive disorder]. Encephale 30: 158-66 
Babiloni C, Lizio R, Marzano N, Capotosto P, Soricelli A, Triggiani Al, Cordone S, Gesualdo L, Del PC (2016) Brain neural synchronization and functional coupling in Alzheimer's disease as revealed by resting state EEG rhythms. Int J Psychophysiol 103: 88-102

Baghai TC, Varallo-Bedarida G, Born C, Hafner S, Schule C, Eser D, Zill P, Manook A, Weigl J, Jooyandeh S, Nothdurfter C, von Schacky C, Bondy B, Rupprecht R (2018) Classical Risk Factors and Inflammatory Biomarkers: One of the Missing Biological Links between Cardiovascular Disease and Major Depressive Disorder. Int J Mol Sci 19

Baskaran A, Farzan F, Milev R, Brenner CA, Alturi S, Pat MM, Blier P, Evans K, Foster JA, Frey BN, Giacobbe P, Lam RW, Leri F, MacQueen GM, Muller DJ, Parikh SV, Rotzinger S, Soares CN, Strother SC, Turecki G, Kennedy SH (2018) The comparative effectiveness of electroencephalographic indices in predicting response to escitalopram therapy in depression: A pilot study. J Affect Disord 227: 542-549

Baskaran A, Milev R, McIntyre RS (2012) The neurobiology of the EEG biomarker as a predictor of treatment response in depression. Neuropharmacology 63: 507-13

Chapotot F, Gronfier C, Jouny C, Muzet A, Brandenberger G (1998) Cortisol secretion is related to electroencephalographic alertness in human subjects during daytime wakefulness. J Clin Endocrinol Metab 83: 4263-8

Chen F, Xu J, Gu F, Yu X, Meng X, Qiu Z (2000) Dynamic process of information transmission complexity in human brains. Biol Cybern 83: 355-66

Delorme A, Makeig S (2004) EEGLAB: an open source toolbox for analysis of single-trial EEG dynamics including independent component analysis. J Neurosci Methods 134: 9-21

Do LLTN (2011) American Psychiatric Association Diagnostic and Statistical Manual of Mental Disorders (DSM-IV)

Faust O, Acharya RU, Allen AR, Lin CM (2008) Analysis of EEG signals during epileptic and alcoholic states using AR modeling techniques. Irbm 29: 44-52

George MS, Ketter TA, Post RM (2010) Prefrontal cortex dysfunction in clinical depression. Depression 2: $59-72$

Hillhouse TM, Porter JH (2015) A brief history of the development of antidepressant drugs: from monoamines to glutamate. Exp Clin Psychopharmacol 23: 1-21

Hlinka J, Alexakis C, Diukova A, Liddle PF, Auer DP (2010) Slow EEG pattern predicts reduced intrinsic functional connectivity in the default mode network: an inter-subject analysis. Neuroimage 53: 239-46

Hunter AM, Cook IA, Leuchter AF (2007) The promise of the quantitative electroencephalogram as a predictor of antidepressant treatment outcomes in major depressive disorder. Psychiatr Clin North Am 30: 
Iznak AF, Sorokin SA (2013) Changes in EEG and Reaction Times during the Treatment of Apathetic Depression. Neurosci Biobehav Physiol43: 79-83

Janjarasjitt S, Scher MS, Loparo KA (2008) Nonlinear dynamical analysis of the neonatal EEG time series: the relationship between neurodevelopment and complexity. Clin Neurophysiol 119: 822-36

Kang JH, Chung YG, Kim SP (2015) An efficient detection of epileptic seizure by differentiation and spectral analysis of electroencephalograms. Comput Biol Med 66: 352-6

Kennedy SH, Andersen HF, Thase ME (2009) Escitalopram in the treatment of major depressive disorder: a meta-analysis. Curr Med Res Opin 25: 161-75

Knyazev GG (2012) EEG delta oscillations as a correlate of basic homeostatic and motivational processes. Neurosci Biobehav Rev 36: 677-695

Kostyalik D, Vas S, Katai Z, Kitka T, Gyertyan I, Bagdy G, Tothfalusi L (2014) Chronic escitalopram treatment attenuated the accelerated rapid eye movement sleep transitions after selective rapid eye movement sleep deprivation: a model-based analysis using Markov chains. BMC Neurosci 15: 120

Lader M, Andersen HF, Baekdal T (2005) The effect of escitalopram on sleep problems in depressed patients. Hum Psychopharmacol 20: 349-54

Li C, Wang J (2008) Similarity analysis of DNA sequences based on the generalized LZ complexity of $(0,1)$-sequences. J Math Chem 43: 26-31

MacGillivray S, Arroll B, Hatcher S, Ogston S, Reid I, Sullivan F, Williams B, Crombie I (2003) Efficacy and tolerability of selective serotonin reuptake inhibitors compared with tricyclic antidepressants in depression treated in primary care: systematic review and meta-analysis. BMJ 326: 1014

Maier W, Buller R, Philipp M, Heuser I (1988) The Hamilton Anxiety Scale: reliability, validity and sensitivity to change in anxiety and depressive disorders. J Affect Disord 14: 61-8

Mikšovský J, Raidl A (2006) Testing for nonlinearity in European climatic time series by the method of surrogate data. Theor Appl Climatol 83: 21-33

Montgomery SA, Baldwin DS, Blier P, Fineberg NA, Kasper S, Lader M, Lam RW, Lepine JP, Moller HJ, Nutt DJ, Rouillon F, Schatzberg AF, Thase ME (2007) Which antidepressants have demonstrated superior efficacy? A review of the evidence. Int Clin Psychopharmacol 22: 323-9

Nofzinger EA, Price JC, Meltzer CC, Buysse DJ, Villemagne VL, Miewald JM, Sembrat RC, Steppe DA, Kupfer DJ (2000) Towards a neurobiology of dysfunctional arousal in depression: the relationship 
between beta EEG power and regional cerebral glucose metabolism during NREM sleep. Psychiatry Res 98: 71-91

Olbrich S, Arns M (2013) EEG biomarkers in major depressive disorder: discriminative power and prediction of treatment response. Int Rev Psychiatry 25: 604-18

Pardey J, Roberts S, Tarassenko L (1996) A review of parametric modelling techniques for EEG analysis. Med Eng Phys 18: 2

Sanchez C, Reines EH, Montgomery SA (2014) A comparative review of escitalopram, paroxetine, and sertraline: Are they all alike? Int Clin Psychopharmacol 29: 185-96

Snaith RP (1977) Hamilton rating scale for depression. Br J Psychiatry 131: 431-2

Stein DJ, Lopez AG (2011) Effects of escitalopram on sleep problems in patients with major depression or generalized anxiety disorder. Adv Ther 28: 1021-37

Steve MG, Bruce A, Simon H, Simon O, lan R, Frank S, Brian W, lain C (2003) Efficacy and tolerability of selective serotonin reuptake inhibitors compared with tricyclic antidepressants in depression treated in primary care: systematic review and meta-analysis. BMJ 326: 1014-1017

Welch PD (1988) The Fast Fourier Transform and Its Applications by E. Oran Brigham.IEEE T Educ12: 2734

\section{Figures}



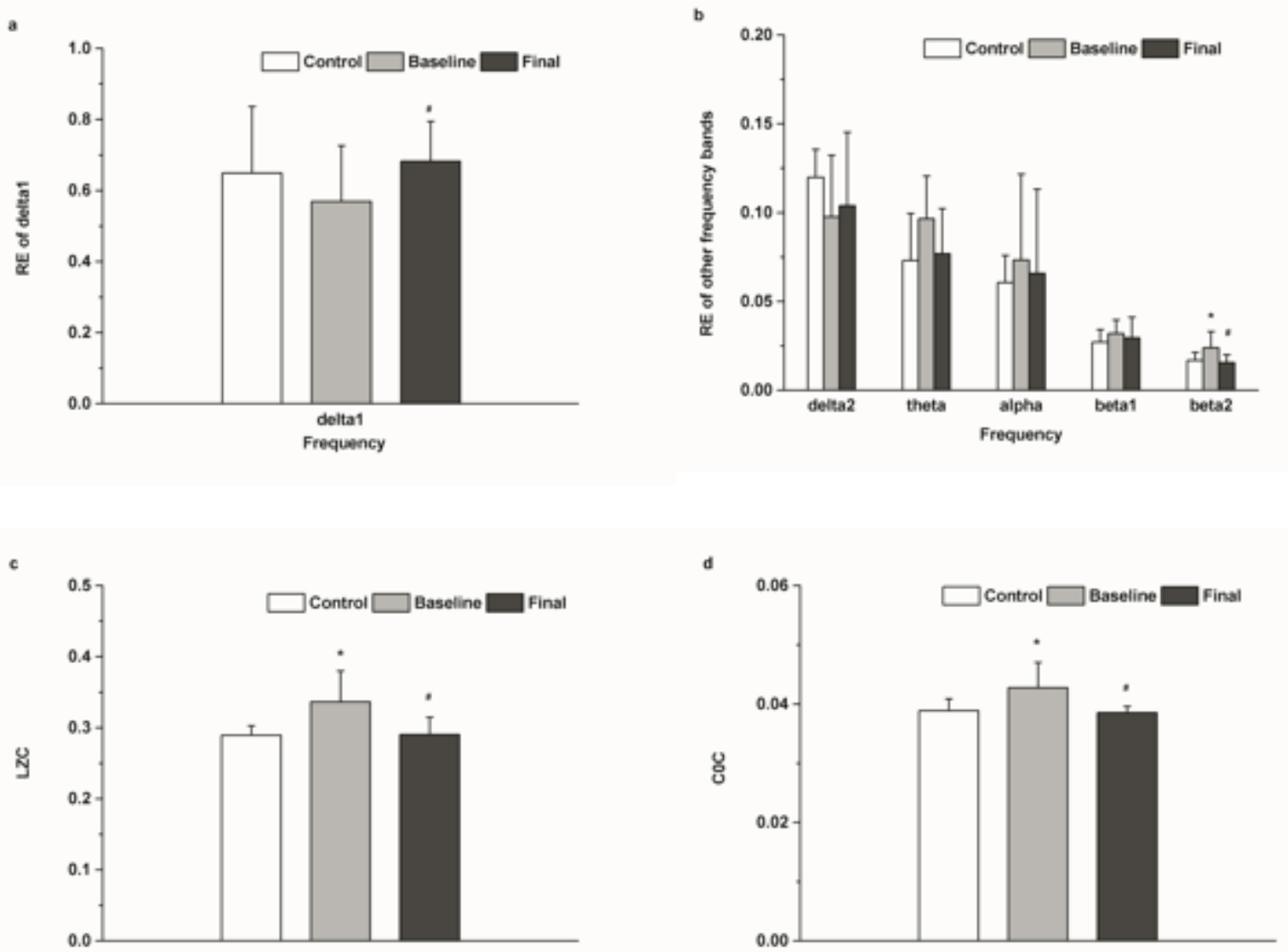

\section{Figure 1}

EEG response before and after escitalopram treatment during the whole sleep process (a) Relative energy (RE) in the $\delta \_1$ band; (b) RE of other frequency bands (except delta1); (c) LZC values; (d) COC values; The data are expressed as mean \pm SD. $n=11$ MDD patients. $n=11$ healthy controls. ${ }^{*} p<0.05$, different from control, \#p < 0.05, different from baseline. 

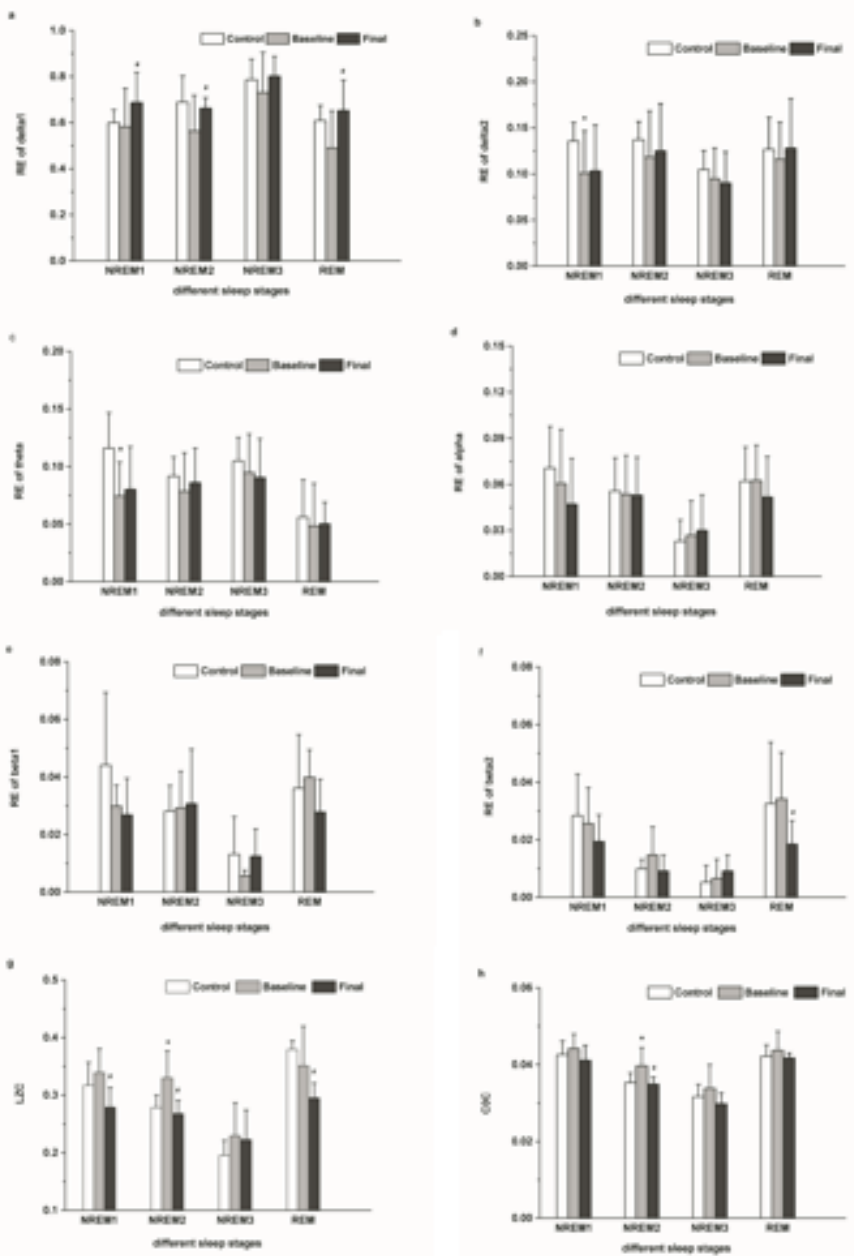

Figure 2

EEG response before and after escitalopram treatment during each sleep stage. (a) Relative energy (RE) in the $\delta \_1$ band; (b) RE in the $\delta \_2$ band; (c) RE in the $\theta$ band; (d) RE in the a band; (e) RE in the $\beta \_1$ band; (f) RE in the $\beta \_2$ band; (g) LZC values; (h) COC values; The data are expressed as mean \pm SD. $n=11 \mathrm{MDD}$ patients. $n=11$ healthy controls. ${ }^{\star} p<0.05$, different from control, $\# p<0.05$, different from baseline. 

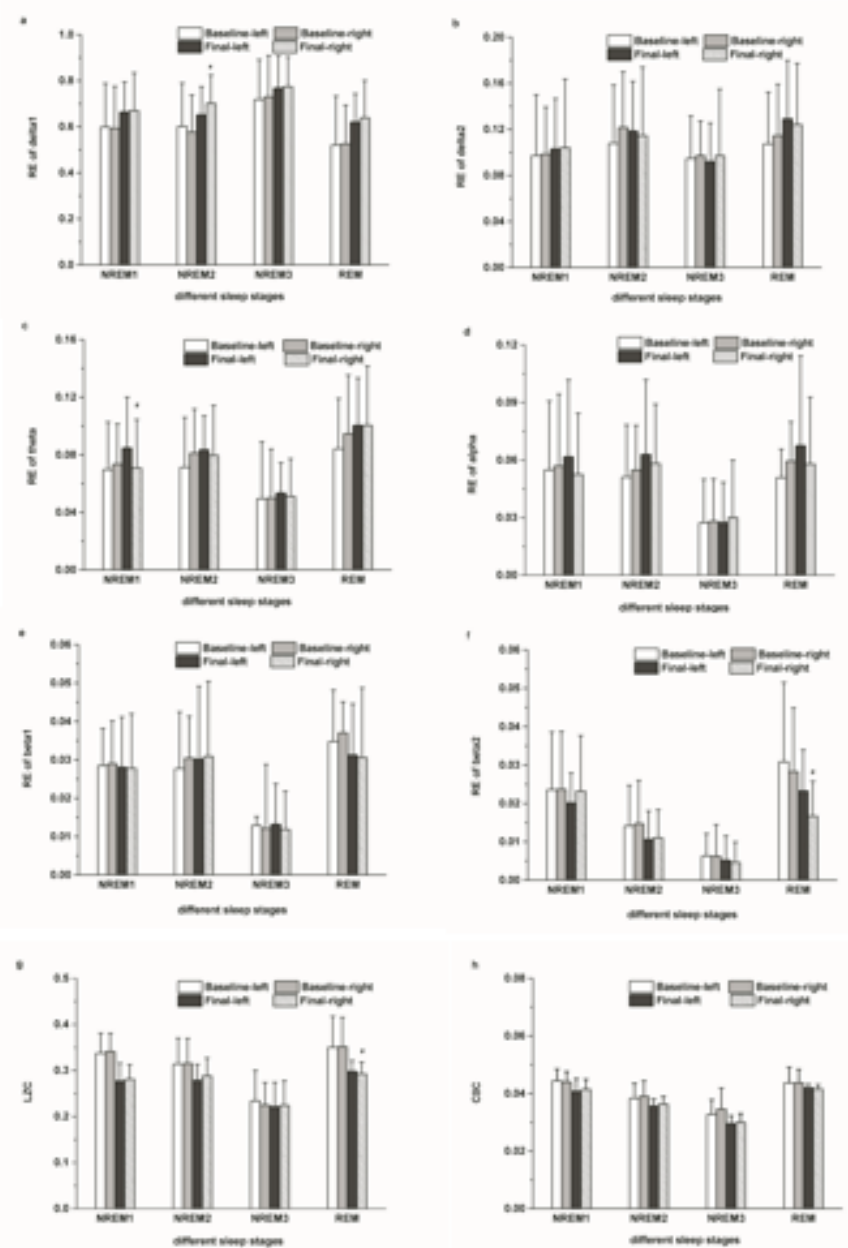

Figure 3

EEG response before and after escitalopram treatment in left and right hemispheres at different sleep stages (a) Relative energy (RE) in the $\delta \_1$ band; (b) RE in the $\delta \_2$ band; (c) RE in the $\theta$ band; (d) RE in the $a$ band; (e) RE in the $\beta \_1$ band; (f) RE in the $\beta \_2$ band; (g) LZC values; (h) COC values; The data are expressed as mean \pm SD. $n=11$ MDD patients. $n=11$ healthy controls. $\# p<0.05$, different from final left.

NREM $1\left(\delta_{1}, \beta_{2}\right.$, LZC $)$

Baseline
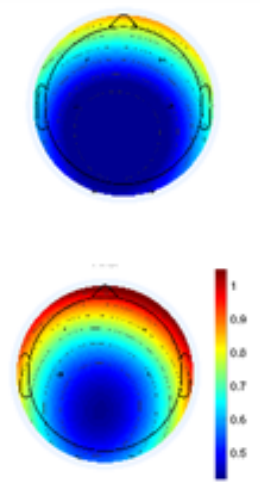

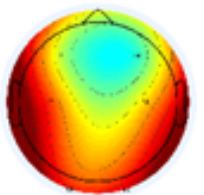

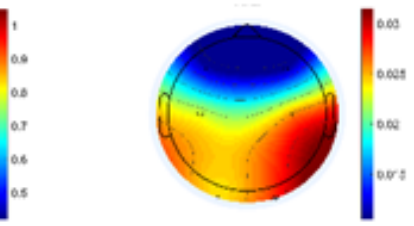

Final
NREM $2\left(\boldsymbol{\delta}_{1}\right)$
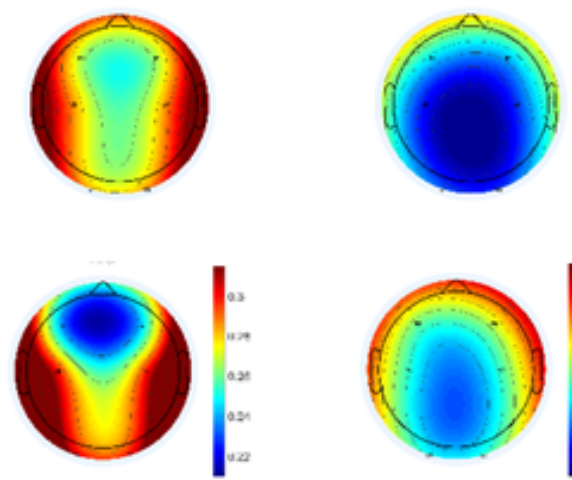

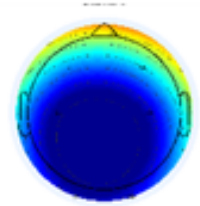


Figure 4

EEG topographic maps of the energy ratio and non-linear LZC values in NREM1, NREM2 and REM before and after treatment. The colors reflect the intensity of the EEG response.

\section{Supplementary Files}

This is a list of supplementary files associated with this preprint. Click to download.

- Methodsformula.docx

- TitlePage.docx 